\title{
External nasal dilation reduces snoring in chronic rhinitis patients: a randomized controlled trial
}

\author{
D. Pevernagie*, E. Hamans**, P. Van Cauwenberge**, R. Pauwels*
}

\begin{abstract}
External nasal dilation reduces snoring in chronic rhinitis patients: a randomized controlled trial. D. Pevernagie, E. Hamans, P. Van Cauwenberge, R. Pauwels. (C)ERS Journals Ltd 2000.

ABSTRACT: Chronic rhinitis patients often suffer from unrefreshing sleep and snoring, related to increased nasal resistance to airflow. Previous trials based on subjective assessment of snoring have demonstrated beneficial effects of Breathe Right $^{\mathrm{TM}}\left(\mathrm{BR}^{\mathrm{TM}}\right)$, a noninvasive external nasal dilator. Polysomnography (PSG) was applied to objectively assess the effects of $B R^{T M}$ on snoring.

Twelve nonobese chronic rhinitis patients participated in the present study, which had a randomized, placebo-controlled design. The presence of snoring and the absence of sleep apnoea was demonstrated during a baseline overnight sleep study. Patients were then randomized for placebo or true nasal dilator treatment, which was also assessed by overnight PSG.

The use of $B R^{\mathrm{TM}}$ had no effect on sleep quality, arousal-index apnoea-hypopnoeaindex or snoring loudness. Snoring frequency was significantly lowered by $B^{\mathrm{TM}}$ $\left(173 \pm 29\right.$ snores $\left.\cdot h^{-1}\right)$, compared with placebo $\left(258 \pm 34\right.$ snores $\left.\cdot h^{-1} ; p=0.016\right)$.

The results support the hypothesis that Breathe Right $^{\mathrm{TM}}$ is effective in reducing the amount of snoring in patients with chronic rhinitis. This objective finding is in line with some other reports on subjective improvement of snoring, based upon the judgement of bedpartners.
\end{abstract}

Eur Respir J 2000; 15: 996-1000.

Depts of *Respiratory Diseases and **Otorhinolaryngology, University Hospital, Ghent, Belgium.

Correspondence: D. Pevernagie, Dept of Respiratory Diseases, University Hospital, De Pintelaan 185, 9000 Ghent, Belgium. Fax. 3292402341

Keywords: Breathe Right ${ }^{\mathrm{TM}}$

external nasal dilation

rhinitis

snoring

Received: June 151999

Accepted after revision March 82000

This research was supported by FWO grant No. 3.0092.93 and by 3M Medical EBC Laboratory, Borken, Germany.
Elevated nasal resistance to airflow is a common finding in patients with mucosal congestion due to chronic rhinitis. Nasal obstruction is associated with an increased frequency of snoring and sleep-disordered breathing. At first, this relationship was suggested in anecdotal reports [1] and reviews of the literature $[2,3]$. In epidemiological surveys it was demonstrated that habitual snoring and sleep disordered breathing is frequently found in patients with chronic rhinitis $[4,5]$.

Snoring typically originates in the pharyngeal segment of the upper airway and is generated by vibrations of soft tissues, including uvula, soft palate, tongue, pharyngeal walls and epiglottis [6]. Snoring characteristics are not only influenced by nasal obstruction, but also by a variety of other factors, e.g. the use of alcohol and sedative drugs, body weight, body posture, and the presence of apnoeic breathing. To reliably assess outcome of a specific therapeutic approach for snoring, adequate control for all these confounders is a prerequisite. Sleep quality and snoring may improve following adequate treatment of nasal obstruction. With the introduction of mechanical nasal dilators in the last decade, an alternative treatment option has become available in addition to surgical or pharmacological approaches. While at first beneficial results with mechanical dilation of the nose were reported [7,8], other investigators found that this particular treatment had limited or no benefits on snoring characteristics [9-11].

Recently, Breathe Right ${ }^{\mathrm{TM}}{ }$ BR $^{\mathrm{TM}} ; 3 \mathrm{M}$, Borken, Germany) has been introduced as a new mechanical device intended to improve nasal breathing. $\mathrm{BR}^{\mathrm{TM}}$ is a disposable noninvasive external nasal dilator. The device consists of an adhesive band containing two elastic springs. When fixed over the bridge of the nose, it dilates the nares by its springboard action, thus decreasing the resistance to airflow at the level of the vestibulum nasi $[12,13]$.

The potential of $\mathrm{BR}^{\mathrm{TM}}$ to reduce snoring and to improve sleep quality by facilitating nasal breathing has been studied in several uncontrolled open label trials [14-16]. Outcome was measured using subjective evaluation tools, mostly questionnaires. Although snoring was reported to improve according to observations of the bedpartners [14, $15]$, and better sleep quality was documented thereafter [16], an important placebo-effect could not be ruled out in each of these trials.

To address the issue of the efficacy of $\mathrm{BR}^{\mathrm{TM}}$ in terms of measurable effects on snoring, the current authors studied patients with chronic rhinitis and nasal obstruction using polysomnography (PSG).

\section{Methods}

Subjects

Subjects were recruited from patients referred for treatment of snoring and volunteers who responded to an advertisement for the study. The selection of patients was based on the following criteria: 1) a history of chronic rhinitis, nasal obstruction and habitual snoring; 2) confirmed nasal obstruction; and 3) confirmed snoring without evidence of significant sleep apnoea. In the course of 10 
months (May 1997-March 1998), 12 eligible individuals were enrolled in the treatment protocol. The participants gave written informed consent to the trial, which was approved by the Ethical Review Board of the institution (University Hospital, Ghent, Belgium). All enlisted patients completed the study.

During the first visit, a detailed history and clinical examination were obtained. Obesity, defined as a body mass index (BMI) of at least $30 \mathrm{~kg} \cdot \mathrm{m}^{-2}$, was an exclusion criterion. Patients who passed the initial screening procedure were referred for ear, nose and throat (ENT) examination. Skin-prick tests were performed to evaluate type I allergy. Oropharyngeal inspection, anterior rhinoscopy and rhinomanometry were performed by the same ENT surgeon. The patients were examined during the daytime under stable conditions representative of their habitual degree of nasal obstruction. None of the participants had acute rhinitis at the time of the ENT evaluation. ENT exclusion criteria included nasal septum deviation, tonsillar hypertrophy, and/or a history of previous ENT surgery. The diagnosis of nasal obstruction was based on a complaint of chronic nasal stuffiness, signs of mucosal oedema revealed by anterior rhinoscopy and elevated nasal resistance. Baseline assessment of nasal conditions was performed only once, at the risk of introducing a selection bias. In the context of the clinical picture, however, a single evaluation of nasal resistance was considered to be appropriate. Active anterior rhinomanometry was carried out in the erect position to measure nasal resistance. An Atmos Rhinomanometer 300 (Atmos, Lenzkirch, Germany) was used to assess pressure/flow relationships separately for each nasal passage during normal tidal breathing. According to international standards [17] nasal resistance is derived from the measurement of the nasal flow rate at a fixed nasal pressure level of $150 \mathrm{~Pa}\left(\mathrm{~Pa} \cdot \mathrm{cm}^{-3} \cdot \mathrm{s}^{-1}\right)$. Elevated nasal resistance was defined as a resistance level of at least 0.5 $\mathrm{Pa} \cdot \mathrm{cm}^{-3} \cdot \mathrm{s}^{-1}$ at one or both sides of the nose. Patients with symptoms and clinical signs of chronic rhinitis in whom an elevated nasal resistance was observed were then scheduled for a baseline sleep study. After baseline measurements, anterior rhinometry was repeated to evaluate the effects of placebo and $\mathrm{BR}^{\mathrm{TM}}$ on nasal resistance.

\section{Sleep studies}

PSG was carried out using a 19-channel digital polygraph (Sleepwalker $^{\mathrm{TM}}$; Medatec, Brussels, Belgium). Electroencephalographic activity from four lead locations (C3-A2, C4-A1, Fz-Cz, Cz-Oz), submental and anterior tibialis electromyographic activity, electro-oculographic activity from two lead locations (left and right eye), as well as electrocardiographic activity from a bipolar chest lead were recorded. Oxyhaemoglobin saturation was measured using pulse-oximetry (Biox $3700^{\mathrm{TM}}$; Ohmeda, Louisville, $\mathrm{CO}$, USA). Oro-nasal airflow was monitored with thermistors. Thoracic and abdominal respiratory movements were recorded using piezo-sensors (Resp-Ez ${ }^{\text {TM}}$; EPM Systems, Midlothian, VA, USA). Posture monitoring was based on a signal derived from a double tilt-switch position sensor attached to the ventral side of the chest. This position sensor discriminated between four recumbent body positions: prone, right side, supine and left side. Only su- pine and nonsupine postures were taken into account for the study of snoring and sleep-disordered breathing.

Data acquisition of snoring comprised two different recording methods which were simultaneously applied. Firstly, a sound signal was derived from a microphonecontaining stethoscope attached to the larynx. The sound was digitized at a sampling rate of $2,000 \mathrm{~Hz}$. This recording technique enabled audio-visual reading of the sound signal and was mainly used for audio-replay in order to differentiate between real snoring and artefacts. Scoring of the snoring events was based on the second method, applied to record the ambient sound level. For this purpose two commercial sound level meters (TES 1350; TES Electrical Electronic Corp., Paipei, Taiwan) were used. The sound measurement was adjusted to a range of 35-100 $\mathrm{dBA}$, with an accuracy of $\pm 2 \mathrm{~dB}$. Calibration was performed using the internal calibration signal of the device at a level of $94 \mathrm{~dB}$. The $\mathrm{dB}$ meters were placed $1 \mathrm{~m}$ from the patient's head at an angle of $90^{\circ}$ with respect to each other. The analogous signals of both devices were monitored continuously by an electronic circuit. The signal of the device with the highest sound level was selected and transmitted to the polygraphic recorder. The purpose of this montage was to control for changes in sound level due to shifting body positions. On screen reading of the digitized signal acquired from the $\mathrm{dB}$ meters allowed the gauging of the peak $\mathrm{dB}$ levels of all individual snores. The background noise level varied between individual recordings (43-45 dB), but within individual recordings the drift of the signal was not more than $\pm 1 \mathrm{~dB}$.

Sleep and respiratory events were manually scored in 30 s epochs. Sleep stages were identified according to standard criteria [18]. The scoring of micro-arousals was based on ad hoc guidelines of the American Sleep Disorders Association [19]. The arousal-index was defined as the total number of micro-arousals divided by total sleep time. Sleep-disordered breathing was defined as the incidence of apnoeas and hypopnoeas. Apnoeas were defined as cessations in airflow lasting for at least $10 \mathrm{~s}$. Hypopnoeas were defined as episodes with an oxyhaemoglobin saturation drop of at least $2 \%$, associated with a qualitative reduction in airflow lasting for at least $10 \mathrm{~s}$. The apnoea-hypopnoea index (AHI) was calculated as the quotient of the total number of apnoeas plus hypopnoeas and total sleep time. AHI was assessed separately for the supine and nonsupine sleep position categories.

Snoring activity was also manually scored in 30 -s pages. Snores were identified on the dB-channel as events with an obvious inspiratory pattern and a peak level of at least $2 \mathrm{~dB}$ above background noise level. When the signal did not indicate unequivocal snoring, the sound of the epoch was replayed and artefacts were discarded. The snoring index (SI) was computed as the ratio of the sum of all the individual snores and total sleep time. In addition, snoring loudness was evaluated by measuring the peak $\mathrm{dB}$ level of all individual snores. Mean snoring loudness across total sleep ( $\mathrm{SN}, \mathrm{dBmax})$ was obtained by averaging the peak $\mathrm{dB}$ values of all snores. Snoring activity was assessed separately for the supine and nonsupine sleeping postures.

These recording methods and scoring rules were applied to all sleep studies. If the first (baseline) sleep study demonstrated significant apnoeic activity (i.e. AHI $\geq 20$ events. $\mathrm{h}^{-1}$ ) or insufficient snoring (i.e. $<15 \%$ of total sleep time), patients were excluded from the trial. 


\section{Trial protocol}

A prospective, randomized, placebo-controlled, doubleblind, crossover study design was applied. $\mathrm{BR}^{\mathrm{TM}}$ and placebo were compared with respect to effect on recorded snoring, which was the primary outcome measure. The placebo was a sham adhesive band with an identical appearance as $\mathrm{BR}^{\mathrm{TM}}$ but without the plastic dilator elements. During a 2nd and 3rd overnight PSG, patients were studied wearing the two different types of nasal auto-adhesive strips in randomized order. Randomization was based on computer-generated pseudo-random numbers. The overnight polysomnograms were carried out on consecutive days in all but two subjects, in whom nasal conditions remained unchanged between the 2nd and 3rd evaluation night. The strips were correctly fixed onto the patients noses by a trained nurse, who was neither allowed to provide information to the investigators, nor involved in outcome assessment. It was verified that the strips were used all night. The patients were not given any information on the quality or supposed action of the strips. The use of sedative drugs was not allowed during the study period. Alcohol intake was prohibited during a time span of $8 \mathrm{~h}$ prior to the commencement of PSG. The investigators and the technologist who scored the polysomnograms were blinded to the study conditions.

\section{Statistical analyses}

In keeping with the cross-over design of the study, repeated measures one-way analysis of variance was performed. Post test analysis was carried out using the Newman-Keuls multiple comparison test, where appropriate. A p-value of $<0.05$ was considered to indicate statistical significance. All presented data are mean \pm SEM.

\section{Results}

Anthropometric and clinical data are shown in table 1. The study cohort consisted of one female and 11 male subjects. Skin tests confirmed type I allergy in four patients. Nasal resistance assessed during wakefulness in the erect body position was lower when $\mathrm{BR}^{\mathrm{TM}}$ was applied $\left(0.74 \pm 0.11 \mathrm{mBar} \cdot \mathrm{L}^{-1} \cdot \mathrm{s}^{-1}\right)$ in comparison with placebo $\left(0.91 \pm 0.14 \mathrm{mBar} \cdot \mathrm{L}^{-1} \cdot \mathrm{s}^{-1}\right)$, the difference being close to statistical significance $(\mathrm{p}=0.06)$.

PSG demonstrated no significant differences between placebo and $\mathrm{BR}^{\mathrm{TM}}$ treatment conditions regarding total sleep time, sleep architecture, sleep fragmentation and AHI (table 2). Data acquisition in the supine body position was

Table 1. - Anthropometric and clinical data

\begin{tabular}{lcc}
\hline & Mean \pm SEM & Range \\
\hline Age yrs & $43 \pm 2.8$ & $25-56$ \\
Length m & $1.74 \pm 0.03$ & $1.50-1.90$ \\
Weight kg & $76.3 \pm 3.8$ & $62-103$ \\
Body mass index kg.m $\mathrm{m}^{-2}$ & $25.1 \pm 0.8$ & $20.6-28.7$ \\
Rhinometry mBar $\cdot \mathrm{L}^{-1} \cdot \mathrm{s}^{-1}$ & $1.07 \pm 0.27$ & $0.52-3.75$ \\
\hline
\end{tabular}

Rhinometry data represent one sided anterior nasal resistance measurements.
Table 2. - Polysomnographic data

\begin{tabular}{lcccc}
\hline & Baseline & Placebo & BR $^{\mathrm{TM}}$ & $\mathrm{p}$-value \\
\hline NREM SL min & $23 \pm 4$ & $22 \pm 9$ & $19 \pm 2$ & $\mathrm{NS}$ \\
REM SL min & $157 \pm 19$ & $118 \pm 15$ & $114 \pm 13$ & $\mathrm{NS}$ \\
TST min & $331 \pm 20$ & $347 \pm 23$ & $365 \pm 20$ & $\mathrm{NS}$ \\
SEF & $74 \pm 4$ & $81 \pm 4$ & $82 \pm 2$ & $\mathrm{p}<0.05$ \\
ST1-2\% & $70 \pm 3$ & $64 \pm 1$ & $65 \pm 3$ & NS \\
ST1-2 min & $228 \pm 13$ & $222 \pm 17$ & $241 \pm 18$ & NS \\
ST3-4\% & $14 \pm 2$ & $17 \pm 3$ & $17 \pm 3$ & NS \\
ST3-4 min & $46 \pm 7$ & $53 \pm 7$ & $56 \pm 7$ & NS \\
STREM \% & $16 \pm 12$ & $20 \pm 12$ & $18 \pm 2$ & NS \\
STREM min & $57 \pm 10$ & $72 \pm 10$ & $68 \pm 10$ & NS \\
AI events $\cdot h^{-1}$ & $29 \pm 3$ & $24 \pm 2$ & $23 \pm 12$ & NS \\
AHI events. ${ }^{-1}$ & $6 \pm 1$ & $7 \pm 2$ & $6 \pm 1$ & NS \\
\hline
\end{tabular}

Values are presented as mean \pm SEM. NREM: nonrapid eye movement; SL: sleep latency; REM: rapid eye movement; TST: total sleep time; SEF: sleep efficiency (=total sleep time/time in bed $\times 100$ ); ST1-2: NREM sleep stage 1 and 2; \%: percentage of total sleep time; ST3-4: NREM sleep stage 3 and 4; STREM: sleep stage REM sleep; AI: arousal-index; AHI: apnoea hypopnoea-index; NS: statistically nonsignificant.

partial since some individuals had no or insufficient sleep time (i.e. $<30 \mathrm{~min}$ ) in that posture during one or more study nights. Therefore, positional effects on sleep disordered breathing could not be analysed. However, six patients had an AHI of $<20$ events. $h^{-1}$ in the supine sleeping position in at least one of the three PSG studies.

Snoring characteristics are presented in figure 1 . The use of $\mathrm{BR}^{\mathrm{TM}}$ had no impact on snoring loudness. SN,dBmax was not different between placebo and $\mathrm{BR}^{\mathrm{TM}}$ treatment conditions $(50.1 \pm 0.6 \mathrm{~dB}$ and $50.1 \pm 0.7 \mathrm{~dB}$, respectively). In contrast, the snoring index decreased significantly during the total sleep period in the $\mathrm{BR}^{\mathrm{TM}}$ treatment condition $\left(173 \pm 29\right.$ snores $\left.\cdot h^{-1}\right)$, as compared with placebo $(258 \pm$ 34 snores $\left.\cdot \mathrm{h}^{-1}, \mathrm{p}=0.016\right)$. The SI was reduced in $10 \mathrm{pa}-$ tients using $\mathrm{BR}^{\mathrm{TM}}$. Supine sleep snoring data could not be analysed due to incomplete data collection. During nonsupine sleep, nasal dilation was associated with a nonsignificant reduction of SI (BR ${ }^{\mathrm{TM}}: 127 \pm 33$ snores $\cdot \mathrm{h}^{-1}$; placebo: $209 \pm 44$ snores $\left.\cdot h^{-1} ; \mathrm{p}=0.07\right)$, and less snoring
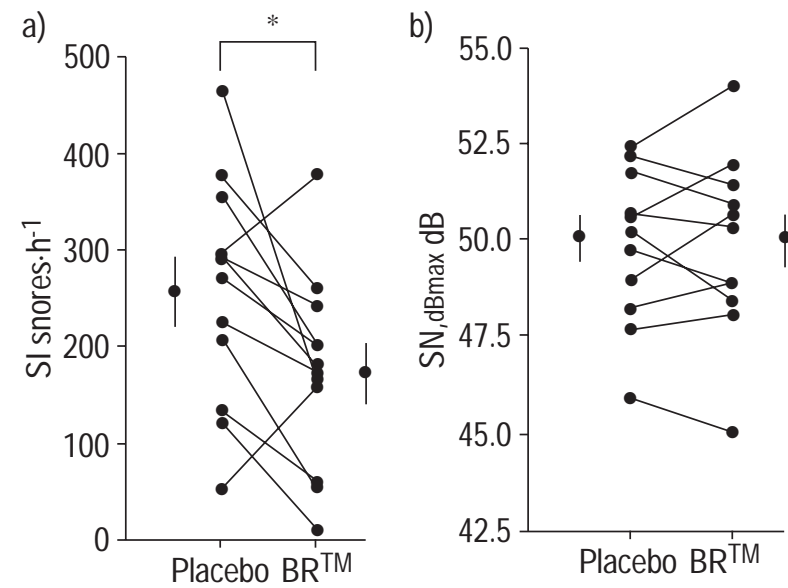

Fig. 1. - Snoring characteristics are presented for total sleep time. Placebo and Breathe Right ${ }^{\mathrm{TM}}\left(\mathrm{BR}^{\mathrm{TM}}\right)$ conditions are compared. Vertical bars represent mean \pm SEM. SI: snoring index $=$ number of snores per hour of sleep; SN,dBmax: average of the peak $\mathrm{dB}$ values of all snores. $*: p=0.016$ (one-way analysis of variance for repeated measures). 
was observed in eight subjects using $\mathrm{BR}^{\mathrm{TM}}$. Snoring loudness was also unaffected by nasal dilation in the nonsupine sleeping posture ( $\mathrm{SN}, \mathrm{dBmax}=49.4 \pm 0.6 \mathrm{~dB}$ in both $\mathrm{BR}^{\mathrm{TM}}$ and placebo treatment conditions).

\section{Discussion}

It was found that $\mathrm{BR}^{\mathrm{TM}}$ had a significant effect on objectively measured snoring in a selected group of nonobese patients with chronic rhinitis and concomitant nasal obstruction, but no other ENT abnormalities. Using BR ${ }^{\mathrm{TM}}$, the snoring index was notably reduced compared with placebo. This effect tended to be maintained in the nonsupine, but could not be evaluated in the supine body position due to incomplete data collection. Contrary to the effects on snoring frequency, the use of $\mathrm{BR}^{\mathrm{TM}}$ did not affect mean snoring loudness.

Although obstructive sleep apnoea (defined as an AHI $\geq 20$ events $\cdot \mathrm{h}^{-1}$ ) was an exclusion criterion in this study, PSG data, including an elevated arousal-index and the presence of positional sleep apnoea in half of the patients, actually indicate that some individuals with sleep disordered breathing were included. In contrast with snoring frequency, which was reduced in 10 out of 12 patients using $\mathrm{BR}^{\mathrm{TM}}$, no significant effect on AHI and arousal-index was observed. Therefore, there is no evidence from the data to show that nasal dilation would affect sleep disordered breathing or concomitant sleep disruption.

The authors assume that the mechanism of the observed effect on snoring is mediated by decreasing nasal resistance during sleep. It was observed that the use of $\mathrm{BR}^{\mathrm{TM}}$ was associated with a small and nearly-significant decrease of nasal resistance in the awake subjects sitting in the upright position, an observation that is in agreement with other publications showing a reduction of nasal resistance following the application of external dilators [12, 13]. However, this hypothesis remains to be proven, since the study protocol did not include the recording of nasal resistance during sleep.

The reason why nasal dilation reduced snoring frequency but not loudness in this group of selected patients also remains elusive. The authors speculate though that a threshold for incipient snoring may be set by nasal conditions: snoring will presumably be initiated once a critical level of increased nasal resistance has been surpassed. However, since vibration is produced at the level of the pharynx, sound characteristics, such as level and pitch, may then become independent from the nose. A reduction in nasal resistance would therefore affect snoring primarily by reducing its appearance and not by altering its sound characteristics.

To the authors knowledge, this is the first randomized controlled trial to demonstrate a reduction of snoring by using a mechanical nasal dilator. PSG has been applied in previous investigations to assess the effects of mechanical nasal dilation on snoring [8-11, 20]. HöIJER et al. [8] studied the effects of Nozovent ${ }^{\mathrm{TM}}$, an internal nasal dilator, on snoring and obstructive sleep apnoea in a group of 11 patients, using a randomized but not controlled study design. They observed that the use of Nozovent ${ }^{\mathrm{TM}}$ was associated with a significant decrease in apnoea frequency and snoring noise, expressed as number of epochs with snores of equal energy levels above 55 or $60 \mathrm{~dB}$. METEs et al. [9] and Hoffstein et al. [10] also used Nozovent ${ }^{\mathrm{TM}}$ in chronic snorers. Although Nozovent ${ }^{\mathrm{TM}}$ was found to substantially reduce nasal resistance during wakefulness, it had no effect on AHI, snoring frequency and maximum sound intensity. Only a reduction of snoring time during slow wave sleep was found [10]. The studies by METES et al. [9] and HoffSTEIN et al. [10] differ from the present trial, not only regarding the study design, which was not controlled nor randomized, but also with respect to inclusion criteria. The patients in the former trials were free of nasal pathology and were found to have nasal resistances within the normal range. Moreover, they were significantly obese (mean \pm SD BMI: $36 \pm 12$ ) [10]. Only nonobese subjects with chronic rhinitis and elevated nasal resistance were included in the present study. Using $\mathrm{BR}^{\mathrm{TM}}$, Listro et al. [11] were unable to detect a reduction of the snoring index in nonapnoeic snorers, and therefore concluded that $\mathrm{BR}^{\mathrm{TM}}$ was ineffective. Again, patients tended to be obese (mean \pm SD BMI: $30 \pm 6$ ) and had no signs of nasal pathology other than undefined nasal valve anomaly. LIISTRO et al. [11] acknowledged the deficient design of their study, which lacked placebocontrol and randomization. TODOROva et al. [20] allowed 30 habitual snorers to spend four consecutive nights in their sleep laboratory. They excluded data from two adaption nights and compared baseline to $\mathrm{BR}^{\mathrm{TM}}$ treatment conditions, using a nonrandomized approach. Out of 37 different snoring parameters, only three were significantly reduced by the $\mathrm{BR}^{\mathrm{TM}}$ treatment condition, namely the maximum snore vibration index for total sleep and stage 1-2 nonrapid eye movement sleep, as well as the number of snores with maximal intensity per hour. The authors failed to observe a reduction of the overall snores per hour index and the overall index of noise. The majority of included subjects had anatomical ENT abnormalities, and some were obese (BMI $\geq 30 \mathrm{~kg} \cdot \mathrm{m}^{-2}$ ).

Whether or not a reduction of snoring is obtained with mechanical nasal dilation, obviously depends on specific patient characteristics. When designing this study, the authors postulated that the potential benefit of $\mathrm{BR}^{\mathrm{TM}}$ could only be demonstrated if its application would be restricted to patients having no other risk factors for snoring than an obstructed nose. The premise that mechanical nasal dilation would affect snoring in obese subjects is not valid from a theoretical point of view. The association between obesity and snoring is to all probability established by the influence of fat accumulation on the pharyngeal airway and not by effects on the nose [21,22]. The significant therapeutic results obtained with $\mathrm{BR}^{\mathrm{TM}}$ in the present study are therefore not in contradiction with previous negative or inconclusive reports, but may be explained by important differences in patients characteristics.

The objective data of this study support the contention that Breathe Right ${ }^{\mathrm{TM}}$ may be proposed as a remedy for snoring in patients with chronic rhinitis. Besides its therapeutic potential, it offers the advantage of having little or no side effects, in contrast with other treatment alternatives aiming to improve nasal patency. Topical decongestants may cause prompt relief of nasal stuffiness, but bear the risk of rebound nasal obstruction and concurrent dependence on the medication [23]. Although surgery may permanently improve nasal patency, the procedure is invasive, requires anaesthesia and has an unpredictable effect on snoring [24]. Therefore Breathe Right ${ }^{\mathrm{TM}}$ may 
prove to be a valid therapeutic choice for snoring in the setting of chronic rhinitis and possibly other causes of impaired nasal breathing.

\begin{abstract}
Acknowledgements. The authors are indebted to all individuals who were involved in the planning and implementation of the study: M. Neyens for the scoring of sleep studies; F. De Poorter for expert patient care (including placement of the nasal dilators); B. Janning, 3M Europe, for supplying "true" and "sham" nasal dilators; Ir. E. Driessens and Ir. S. Adams, Medatec, Belgium, for adapting the polysomnographic equipment to the experimental snoring protocol. Last but not least, the authors wish to thank the patients who were willing to participate.
\end{abstract}

\section{References}

1. Lavie P. Nasal obstructions, sleep and mental function. Sleep 1983; 6: 244-246.

2. Olsen KD, Kern EB. Nasal influences on snoring and obstructive sleep apnea. Mayo Clin Proc 1990; 65: 10951105.

3. Papsidero MJ. The role of nasal obstruction in obstructive sleep apnea syndrome. Ear Nose Throat J 1993; 72: 8284.

4. Young T, Finn L, Kim H, et al. Nasal obstruction as a risk factor for sleep-disordered breathing. J Allergy Clin Immunol 1997; 99: S757-S762.

5. McNicholas WT, Tarlo S, Cole P, et al. Obstructive apneas during sleep in patients with seasonal allergic rhinitis. Am Rev Respir Dis 1982; 126: 625-628.

6. Hoffstein V. Snoring. Chest 1996; 109: 201-222.

7. Petruson B, Theman K. Clinical evaluation of the nasal dilator Nozovent. The effect on snoring and dryness of the mouth. Rhinology 1992; 30: 283-287.

8. Höijer U, Ejnell H, Hedner J, Petruson B, Eng LB. The effects of nasal dilation on snoring and obstructive sleep apnea. Arch Otolaryngol Head Neck Surg 1992; 118: 281-284.

9. Metes A, Cole P, Hoffstein V, Miljeteig H. Nasal airway dilation and obstructed breathing in sleep. Laryngoscope 1992; 102: 1053-1055.
10. Hoffstein V, Mateika S, Metes A. Effect of nasal dilation on snoring and apneas during different stages of sleep. Sleep 1993; 16: 360-365.

11. Liistro G, Rombaux P, Dury M, Pieters T, Aubert G, Rodenstein DO. Effects of Breathe Right ${ }^{\mathrm{TM}}$ on snoring: a polysomnographic study. Respir Med 1998; 92: 10761078.

12. Gosepath J, Mann WJ, Amedee RG. Effects of the breathe right nasal strips on nasal ventilation. Am J Rhinol 1997; 11: 399-402.

13. Griffin JW, Hunter G, Ferguson D, Sillers MJ. Physiologic effects of an external nasal dilator. Laryngoscope 1997; 107: 1235-1238.

14. Scharf MB, Brannen DE, McDannold M. A subjective evaluation of a nasal dilator on sleep \& snoring. Ear Nose Throat J 1994; 73: 395-401.

15. Ulfberg J, Fenton G. Effect of Breathe Right nasal strip on snoring. Rhinology 1997; 35: 50-52.

16. Scharf MB, McDannold MD, Zaretsky NT, Hux GT, Brannen DE, Berkowitz DV. Cyclic alternating pattern sequences in non-apneic snorers with and without nasal dilation. Ear Nose Throat J 1996; 75: 617-619.

17. Clement P. Committee report on standardization of rhinomanometry. Rhinology 1984; 22: 151-155.

18. Rechtschaffen A, Kales A. A manual of standardized terminology, techniques and scoring system for sleep stages of human subjects. U.S. Government Printing Office, Washington DC, USA, 1968.

19. American sleep disorders association. EEG arousals: scoring rules and examples. A preliminary report from the atlas task force of the American Sleep Disorders Association. Sleep 1992; 15: 173-184.

20. Todorova A, Schellenberg R, Hofmann HC, Dimpfel W. Effect of the external dilator Breathe Right on snoring. Eur J Med Res 1998; 3: 367-379.

21. Shelton KE, Woodson H, Gay S, Suratt PM. Pharyngeal fat in obstructive sleep apnea. Am Rev Respir Dis 1993; 148: $462-466$.

22. Hoffstein V, Zamel N, Phillipson EA. Lung volume dependence of pharyngeal cross-sectional area in patients with obstructive sleep apnea. Am Rev Respir Dis 1984; 130: $175-178$.

23. Graf P. Rhinitis medicamentosa: aspects of pathophysiology and treatment. Allergy 1997; 52: 28-34.

24. Fairbanks DN. Effect of nasal surgery on snoring. South Med J 1985; 78: 268-270. 